\title{
The evolving clinical picture of chronic prostatitis/chronic pelvic pain syndrome (CP/CPPS): A look at 1310 patients over 16 years
}

\author{
R. Christopher Doiron ${ }^{1}$; Dean Tripp ${ }^{1,2}$; Victoria Tolls ${ }^{1}$; J Curtis Nickel ${ }^{1}$ \\ ${ }^{1}$ Department of Urology, Queen's University; ${ }^{2}$ Department of Psychology, Queen's University, Kingston, \\ ON, Canada
}

Acknowledgements: Dr. Nickel is supported by grants from the CIHR and NIH/NIDDK.

Cite as: Can Urol Assoc J 2018 Feb. 23; Epub ahead of print.

http://dx.doi.org/10.5489/cuaj.4876

Published online February 23, 2018

\section{$* * *$}

\section{Abstract}

Introduction: Two decades of increasing understanding of etio-pathogenesis and clinical phenotyping produces an impression the clinical face of chronic prostatitis/chronic pelvic pain syndrome (CP/CPPS) is changing. We sought to retrospectively analyze trends in CP/CPPS patients presenting to our clinic for evaluation over a 16-year period.

Methods: Patients with CP/CPPS presenting to a tertiary clinic were evaluated prospectively from 1998-2014 with chronic prostatitis symptom index (CPSI) and UPOINT categorization. Patients were stratified in four cohorts, based on year of presentation, and we retrospectively analyzed variations in symptom scores and patterns, UPOINT categorization, and treatment modalities amongst cohorts.

Results: Mean age of the 1310 CP/CPPS patients was 44.7 years, while mean CPSI pain, urination, and total scores were 10.6, 4.8, and 23.3, respectively. The most prevalent UPOINT domain, urinary (U) (71.8\%) was associated with a higher CPSI urination score (6.3), more frequent penile tip pain (37\%), dysuria (48\%), and more treatment with alpha-blockers (70\%). Increase in UPOINT domains was associated with higher CPSI pain, quality of life (QoL), and total scores. Trends over time included increased prevalence of psychosocial (P), organ (O) and tenderness $(\mathrm{T})$ domains, as well as increased use of alpha-blockers, neuromodulation, and phytotherapy as treatment modalities. There was little variation in age, CPSI scores, and pain locations over time. Conclusions: The changing clinical face of CP/CPPS reflects the increased recognition of psychosocial (P domain) and pelvic floor pain ( $\mathrm{T}$ domain), along with the concomitant 
use of associated therapies. There was little variation of pain/urinary symptom patterns and QoL.

\section{Introduction}

Prostatitis syndromes including prostate pain complaints amongst men have long been recognized as a common clinical scenario encountered by physicians. ${ }^{1,2}$ It took several years of concerted effort from the National Institutes of Health (NIH) via their Chronic Prostatitis Collaborative Research Network (CPCRN) to help define the disease of Chronic Prostatitis/Chronic Pelvic Pain Syndrome (CP/CPPS). International consensus following a 1995 Chronic Prostatitis workshop classified CP/CPPS into 4 categories $^{3,4}$ this helped define the disease for clinicians, provided a framework from which to approach a disease with a challenging, heterogeneous presentation, and furthermore, allowed for a more standardized approach to research in the field.

The continued enthusiasm of the NIH collaboration led to the development of a validated symptom index tool ${ }^{5}$ - the NIH Chronic Prostatitis Symptom Index (CPSI) that has allowed for objective evaluation of patients and their response to treatment. This tool has proved invaluable in evaluating various treatments for CP/CPPS in clinical trials, ${ }^{6-8}$ has proved helpful in evaluating CP/CPPS prevalence ${ }^{9}$ and has been used to validate tiers of disease severity. ${ }^{10}$

Despite these advancements, CP/CPPS remained a difficult entity to manage. Randomized controlled trials of various treatment modalities failed to show significant improvements in measured outcomes. ${ }^{11}$ Furthermore, the etiology and pathogenesis of $\mathrm{CP} / \mathrm{CPPS}$ remained an enigma and likely represented a constellation of disease contributors and pathways. There were, however, some subgroups that appeared to benefit from various treatment modalities. ${ }^{11,12}$ It was becoming accepted that the heterogeneous nature of patients presenting with CP/CPPS would be best evaluated by subgrouping patients according to either mechanisms, biomarkers or symptoms. Using available clinical assessment, a phenotypic approach to classifying patients in clinical practice was proposed. UPOINT phenotyping ${ }^{13}$ built on the improvements made by the NIH classification by further recognizing even within these 4 broad categories, patients were still heterogeneous. Assessing individual patients according to the proposed six domains allowed for improved patient-centered care, leading to development of individualized clinical treatment strategies. ${ }^{14}$

It has now been close to two decades since our updated classification system came into general use, a decade since UPOINT was first introduced and this has led to a plethora of current research in the field. But have any of these developments made an impact on patient evaluation and subsequent treatment?

This study is a retrospective look at CP/CPPS patients evaluated in a single outpatient clinic over the course of a sixteen-year period, beginning with introduction of 
our current classification system and spanning the introduction of UPOINT phenotyping. We were interested in observing the initial presentation of CP/CPPS patients in a tertiary prostatitis clinic to determine changes in symptom patterns, UPOINT phenotype, and treatment modality trends over time.

\section{Methods}

Participants and study design

This study is a retrospective examination of a cohort of CP/CPPS patients examined at a single outpatient clinic. The cohort was examined as part of a large prospective clinical quality assurance database and was examined by a single urologist (JCN). The evaluation of this patient population has been described in previous publications. ${ }^{6}$ Data presented was collected from initial presentation between the years 1998-2014. This study was done under ongoing IRB approval for continued quality assurance with all patient data de-identified before analysis.

\section{Measures}

Data regarding the patients’ demographics, symptom duration, CPSI scores (pain, urinary, impact/quality of life), UPOINT scoring were collected through initial evaluations at the CP/CPPS outpatient clinic (UPOINT scoring was retrospective prior to 2009 and prospective from 2009). Data regarding treatments received therefore represent interventions prior to initial evaluation at CP/CPPS clinic and would have been administered by referring physicians. Data was examined first as an overall cohort and then over time in 4 separate cohorts ranging from 1998-2001, 2003-2005, 2006-2009, and 2010-2014.

\section{Data analysis}

Statistical analysis was completed using Microsoft Excel 2010 Data Analysis package and the online Social Science Statistics software (http://www.socscistatistics.com). Continuous variables (age and CPSI scores) were analyzed using ANOVA (using R version 3.3.1) with $\mathrm{p}<0.05$ regarded as significant. Categorical variables (UPOINT, pain locations and treatments) were analyzed using Chi-squared Test for Trend in Proportions (using $\mathrm{R}$ version 3.3.1), with $\mathrm{p}<0.05$ regarded as significant.

\section{Results}

Between the years of 1998-2014, 1349 patients with CP were evaluated at a single tertiary referral outpatient clinic. Of these, 39 patients were diagnosed with bacterial prostatitis (Category II) and were excluded from the current analysis. The remaining 1310 patients were given a diagnosis of Category III CP/CPPS. The majority of patients $(n=804,61.4 \%)$ were referred by a general practitioner, while 474 (36.2\%) patients were referred by another Urologist. Mean age of the 1310 CP/CPPS patients was 44.7 years 
(Table 1). Their mean CPSI pain, urination, QOL and total scores were 10.6, 4.8, 7.9 and 23.3 respectively (Table 1 ).

Overall, the most prevalent UPOINT domain was the urinary ("U”) domain ( $\mathrm{n}=941,71.8 \%$ ), followed by organ-specific (“O”) ( $\mathrm{n}=660,50.4 \%)$, tenderness (“T”) ( $n=644,49.2 \%)$, infection (“I”) ( $n=440,33.6 \%)$, neurologic (“N”) (n=402, 30.7\%), and psychosocial ("P”) ( $n=361,27.6 \%)$ domains (Table 1). Positive reporting of the urinary domain was associated with a higher CPSI total score (25.8), higher CPSI urination score (6.3), more frequent penile tip pain ( $n=348,37 \%)$, more frequent dysuria $(n=454,48.2 \%)$, and more were more commonly treated with alpha-blockers ( $\mathrm{n}=659,70 \%)$ (Appendix 1). Those patients who reported in the psychosocial domain ("P") were associated with a higher UPOINT total score (3.3) and were more commonly treated with antidepressants ( $n=105,29.1 \%$ ) and gabapentinoids $(n=37,10.2 \%)$ compared with the other domains (Appendix 1).

An increased reporting of total UPOINT domains was associated with higher CPSI pain score (14.3), worse QOL (10.9) and higher total CPSI scores (31.4) (Appendix 2). Pain location was not associated with severity of disease, nor was it associated with UPOINT domain or treatment modality (Appendix 3).

An analysis of trends over time through examination of the 4 separate time cohorts revealed an increased prevalence of psychosocial domain ("P”) ( $\mathrm{p}=0.0009)$, organ domain ("O") $(\mathrm{p}=<0.0001)$ and tenderness domain ("T") $(\mathrm{p}=<0.0001)$ (Table 2). A decrease in the infection ("I") $(\mathrm{p}=<0.0001)$ over time was also noted (Table 2$)$. In terms of treatment modalities, there was an increased prevalence of use of alpha-blockers $(\mathrm{p}=<0.0001)$, neuromodulation $(\mathrm{p}=<0.0001)$ and phytotherapy $(\mathrm{p}=0.0001)$ with time, while the use of antibiotics - which ranged from $20.5 \%-27.2 \%$ amongst the cohorts did not change significantly with time (Table 2). There was little variation in age, CPSI scores and pain locations over time.

\section{Discussion}

We describe the change in specific CP/CPPS symptoms (CPSI scoring), clinical phenotypes (as expressed by UPOINT), and the correlation between these parameters over time in this retrospective study of $1310 \mathrm{CP} / \mathrm{CPPS}$ patients, presenting to a single clinic over a 16 year period. The analysis confirmed that an increased number of UPOINT domains was associated with increased CPSI scores as previously suggested by Shoske et al. ${ }^{12}$ It further showed that referring physicians tailored treatments to clinical presentation before UPOINT was described. It appears that both before and after UPOINT introduction physicians were treating patients with urinary symptoms (" $U$ " domain) with alpha-blockers, using antidepressants with secondary pain modulation in patients with psychological problems ("P” domain), neuromodulatory medications (gabapentinoids) for neurogenic pain (“ $\mathrm{N}$ ” domain), and increasing use of physiotherapy 
for pelvic floor pain (“T” domain). Although many patients - particularly in the earlier cohorts - were identified with infection at some time in their past ("I" domain), our observation of a trend of less antibiotics being employed over time for these patients was compatible with increased understanding of the inappropriateness of antimicrobial therapy in the majority of CP/CPPS patients.

We were surprised that patient identified pain locations (CPSI) did not necessarily correlate with total CPSI score, UPOINT domains or treatment. This is in keeping with a large multinational study of 1563 CP/CPPS patients by Wagenlehner et al ${ }^{10}$ where they found that pain severity and frequency were more important than pain location. CP/CPPS is a heterogeneous disease and pain location may not be overly useful in helping direct treatment i.e. pain is pain, regardless of location.

We observed a number of trends in UPOINT domain prevalence over time, specifically an increase over time in the "P" and “ $\mathrm{T}$ ” domains. This likely does not reflect a change in patient population presenting characteristics, but rather our referring physicians' understanding of the importance of identifying these domains for better treatment outcomes. In fact, it appears that the patients are not changing over time. The age, CPSI score (and pain, urinary and impact/quality of life sub-scores) and pain locations did not appreciably change from 1998-2014.

The low use of antibiotics (22.4\% overall) was interesting to note, given that current guidelines, acknowledging the generally poor evidence for use in category III CP/CPPS patients, recommend or suggest consideration of a course of antimicrobial therapy as a first-line treatment option. ${ }^{15-17}$ Although no trends in antibiotic use were seen over time, their relatively low usage as a treatment modality may reflect an increased attention to antimicrobial stewardship amongst general practitioners. This is in contrast to the increased trend over time in usage of neuromodulation and phytotherapy. This could similarly reflect an increased comfort amongst general practitioners with neuromodulatory medication prescribing, while increased use of phytotherapy may illustrate a patient population increasingly interested in seeking alternative health strategies and natural products.

While it is encouraging to note that referring physicians appeared to be embracing specific treatments towards identified phenotypes prior to referral to our specialty clinic, it is difficult to know if this has made a significant difference to patient outcome. Certainly, even with the described treatment identified in this referral population, baseline symptoms were similar to those of patients being enrolled in clinical treatment trials. ${ }^{11}$ Further research will attempt to determine whether identification of specific phenotypes along with assessment of failed therapies resulted in therapeutic strategies that provided favorable patient outcomes by examining this same patient population one year after the first clinic assessment described in the present study. 


\section{Conclusions}

The increased recognition of the psychosocial ("P" domain) and pelvic floor pain ("T" domain) by referring physicians may be responsible for the changing clinical face of $\mathrm{CP} / \mathrm{CPPS}$. This increased recognition of specific domains coincides with increased use of their associated therapies. However, there was little change in pain or urinary symptom patterns and QOL over the 16 years of assessment. The more things “appear” to change, the more they stay the same. 


\section{References}

1. Thin RN. The diagnosis of prostatitis: a review. Genitourin Med 1991; 67: 279-83.

2. Roberts RO, Lieber MM, Bostwick DG, et al. A review of clinical and pathological prostatitis syndromes. Urology 1997; 49: 809-21.

3. Krieger JN, Nyberg L Jr, Nickel JC. NIH consensus definition and classification of prostatitis. JAMA 1999; 282: 236-37.

4. Nickel JC, Nyberg LM, Hennenfent M. Research guidelines for chronic prostatitis: consensus report from the First National Institutes of Health International Prostatitis Collaborative Network. Urology 1999; 54: 229-33.

5. Litwin MS, McNaughton-Collins M, Fowler FJ Jr, et al. The National Institutes of Health chronic prostatitis symptom index: development and validation of a new outcome measure. Chronic Prostatitis Collaborative Research Network. J Urol 1999; 162: 369-75. 6. Wagenlehner FME, Schneider H, Ludwig M, et al. A pollen extract (Cernilton) in patients with inflammatory chronic prostatitis-chronic pelvic pain syndrome: a multicentre, randomised, prospective, double-blind, placebo-controlled phase 3 study. Eur Urol 2009; 56: 544-51.

7. Nickel JC, Pontari M, Moon T, et al. A randomized, placebo controlled, multicenter study to evaluate the safety and efficacy of rofecoxib in the treatment of chronic nonbacterial prostatitis. J Urol 2003; 169: 1401- 5.

8. Nickel JC, Krieger JN, McNaughton-Collins M, et al. Alfuzosin and symptoms of chronic prostatitis-chronic pelvic pain syndrome. N Engl J Med 2008; 359: 2663-73. 9. Nickel JC, Downey J, Hunter D, et al. Prevalence of Prostatitis-like Symptoms in a Population-based Study using the National Institutes of Health Chronic Prostatitis Symptom Index. J Urol 2001; 165; 842-45.

10. Wagenlehner FME, van Till JWO, Magri V, et al. National Institutes of Health Chronic Prostatitis Symptom Index (NIH-CPSI) Symptom Evaluation in Multinational Cohorts of Patients with Chronic Prostatitis/Chronic Pelvic Pain Syndrome. Eur Urol 2013; 63: 953-9.

11. Anothaisintawee T, Attia J, Nickel JC, et al. The Management of Chronic Prostatitis/Chronic Pelvic Pain Syndrome: A systematic review and network metaanalysis. JAMA 2011; 305: 78-86.

12. Shoskes DA, Nickel JC, Dolinga R, Prots D. Clinical Phenotyping of Chronic Prostatitis/Chronic Pelvic Pain Patients and Correlation with Symptom Severity. Urology 2009; 73: 538-43.

13. Nickel JC, Shoskes D: Phenotpyic Approach to the Management of Chronic Prostatitis/Chronic Pelvic Pain Syndrome. D. Curr Urol Rep 2009; 10: 307-12. 
14. Shoskes D, Nickel JC, Rackley RR, et al. Clinical phenotyping in chronic prostatitis/chronic pelvic pain syndrome and interstitial cystitis: a management strategy for urologic chronic pelvic pain syndromes. Prostate Cancer Prostatic Dis 2009; 12: 177-83.

15. Nickel JC. Prostatitis. Can Urol Assoc J 2011; 5: 306-15.

16. Rees J, Abrahams M, Doble A, et al. Diagnosis and treatment of chronic bacterial prostatitis and chronic prostatitis/chronic pelvic pain syndrome: a consensus guideline. BJU Int 2015; 116: 509-25.

17. Sharp VJ, Takacs EB, Powell CR. Prostatitis: Diagnosis and Treatment. Am Fam Physician 2010; 82: 397-406. 


\section{Figures and Tables}

\section{Table 1. Age, CPSI scores, UPOINT analysis, pain locations, and treatments provided by referring physicians for $1310 \mathrm{CP} / \mathrm{CPPS}$ patients evaluated in a single outpatient clinic from 1998-2014}

\begin{tabular}{|c|c|}
\hline & 1998-2014 \\
\hline & $n=1310$ \\
\hline Age & $44.7( \pm 13.3)$ \\
\hline CPSI pain & $10.6( \pm 4.9)$ \\
\hline CPSI urination & $4.8( \pm 3.1)$ \\
\hline CPSI QoL & $7.9( \pm 3.1)$ \\
\hline CPSI total & $23.3( \pm 9.0)$ \\
\hline UPOINT & \\
\hline $\mathrm{U}$ & $941(71.8 \%)$ \\
\hline $\mathrm{P}$ & $361(27.6 \%)$ \\
\hline $\mathrm{O}$ & $660(50.4 \%)$ \\
\hline $\mathrm{I}$ & $440(33.6 \%)$ \\
\hline $\mathrm{N}$ & $402(30.7 \%)$ \\
\hline $\mathrm{T}$ & $644(49.2 \%)$ \\
\hline Pain location & \\
\hline Perineum & $788(60.2 \%)$ \\
\hline Testicular & $658(50.2 \%)$ \\
\hline Tip of penis & $441(33.7 \%)$ \\
\hline Pubic/bladder & 798 (60.9\%) \\
\hline Urination & $553(42.2 \%)$ \\
\hline Ejaculation & 615 (46.9\%) \\
\hline Treatment by referring physi & \\
\hline Alpha-blockers & 806 (61.5\%) \\
\hline Antidepressants & $198(15.1 \%)$ \\
\hline Quercetin & $449(34.3 \%)$ \\
\hline QUrol & $115(8.8 \%)$ \\
\hline Gabapentinoids & $79(6.0 \%)$ \\
\hline Acupuncture/acupressure & $10(0.8 \%)$ \\
\hline Physiotherapy & $33(2.5 \%)$ \\
\hline Antibiotics & $293(22.4 \%)$ \\
\hline Prostate massage & $43(3.3 \%)$ \\
\hline
\end{tabular}

${ }^{*}$ Refers to treatment provided by referring physician prior to or at time of evaluation of presentation to chronic prostatitis clinic. CPSI: chronic prostatitis symptom index; QoL: quality of life. 


\section{Table 2. Trends observed over time in $1310 \mathrm{CP} / \mathrm{CPPS}$ patients stratified by time of initial presentation to chronic prostatitis clinic}

\begin{tabular}{|c|c|c|c|c|c|}
\hline & 1998-2001 & 2002-2005 & 2006-2009 & 2010-2014 & \multirow[t]{2}{*}{$\mathbf{p}$} \\
\hline & $n=195$ & $n=387$ & $\mathrm{n}=357$ & $\mathrm{n}=371$ & \\
\hline Age & $42.1( \pm 11.3)$ & $44.9( \pm 12.3)$ & $45.8( \pm 13.2)$ & $44.9( \pm 15.3)$ & 0.0185 \\
\hline CPSI pain & $11.1( \pm 4.6)$ & $10.6( \pm 4.6)$ & $10.2( \pm 5.1)$ & $10.7( \pm 5.0)$ & 0.2000 \\
\hline CPSI urination & $4.5( \pm 2.9)$ & $5.1( \pm 3.0)$ & $4.6( \pm 3.1)$ & $5.0( \pm 3.3)$ & 0.0564 \\
\hline CPSI QoL & $7.8( \pm 3.0)$ & $7.7( \pm 3.0)$ & $7.8( \pm 3.1)$ & $8.2( \pm 3.2)$ & 0.2272 \\
\hline CPSI total & $23.4( \pm 8.3)$ & $23.4( \pm 8.8)$ & $22.7( \pm 9.2)$ & $23.8( \pm 9.3)$ & 0.3599 \\
\hline \multicolumn{6}{|l|}{ UPOINT } \\
\hline $\mathrm{U}$ & $144(73.8 \%)$ & $291(75.2 \%)$ & $250(70.0 \%)$ & $256(69.0 \%)$ & 0.0636 \\
\hline $\mathrm{P}$ & $45(23.1 \%)$ & $94(24.3 \%)$ & $94(26.3 \%)$ & $128(34.5 \%)$ & 0.0009 \\
\hline $\mathrm{O}$ & 67 (34.4\%) & $175(45.2 \%)$ & $224(62.7 \%)$ & $194(52.3 \%)$ & $<0.0001$ \\
\hline $\mathrm{I}$ & $116(59.5 \%)$ & $140(36.2 \%)$ & $90(25.2 \%)$ & $94(25.3 \%)$ & $<0.0001$ \\
\hline $\mathrm{N}$ & $88(45.1 \%)$ & $115(29.7 \%)$ & $95(26.6 \%)$ & $104(28.0 \%)$ & 0.0003 \\
\hline $\mathrm{T}$ & $55(28.2 \%)$ & $173(44.7 \%)$ & $156(43.7 \%)$ & $260(70.1 \%)$ & $<0.0001$ \\
\hline \multicolumn{6}{|l|}{ Pain locations } \\
\hline Perineum & $138(70.8 \%)$ & $230(59.4 \%)$ & $210(58.8 \%)$ & $210(56.6 \%)$ & 0.0049 \\
\hline Testicular & 95 (48.7\%) & 215 (55.6\%) & $163(45.7 \%)$ & 185 (49.9\%) & 0.3924 \\
\hline Tip of penis & $57(29.2 \%)$ & 137 (35.4\%) & $115(32.2 \%)$ & 132 (35.6\%) & 0.3195 \\
\hline Pubic/bladder & $121(62.1 \%)$ & $246(63.6 \%)$ & 217 (60.8\%) & $214(57.7 \%)$ & 0.1456 \\
\hline Dysuria & $92(47.2 \%)$ & 165 (42.6\%) & $146(40.9 \%)$ & $150(40.4 \%)$ & 0.1368 \\
\hline Ejaculation & $104(53.3 \%)$ & $182(47.0 \%)$ & 165 (46.2\%) & $164(44.2 \%)$ & 0.0591 \\
\hline \multicolumn{6}{|c|}{ Treatment by referring physician $^{*}$} \\
\hline Alpha-blockers & $64(32.8 \%)$ & $244(63 \%)$ & $263(73.7 \%)$ & 235 (63.3\%) & $<0.0001$ \\
\hline Neuromodulation & $16(8.2 \%)$ & $45(11.6 \%)$ & 79 (22.1\%) & 95 (25.6\%) & $<0.0001$ \\
\hline Phytotherapy & $62(31.8 \%)$ & $139(35.9 \%)$ & $204(57.1 \%)$ & 159 (42.9\%) & 0.0001 \\
\hline Physiotherapy & $13(6.6 \%)$ & $21(5.4 \%)$ & $13(3.6 \%)$ & 36 (9.7\%) & 0.1078 \\
\hline Antibiotics & $53(27.2 \%)$ & $82(21.2 \%)$ & $82(23 \%)$ & 76 (20.5\%) & 0.1759 \\
\hline
\end{tabular}

${ }^{*}$ Refers to treatment provided by referring physician prior to or at time of evaluation of presentation to chronic prostatitis clinic. CP/CPPS: chronic prostatitis/chronic pelvic pain syndrome; CPSI: chronic prostatitis symptom index; QoL: quality of life. 